\title{
Envolvimento sistêmico da doença periodontal - Quais as consequências?
}

\author{
Renata Diniz SOUZA ${ }^{\mathrm{I}}$ \\ Kétlin Karen de ANDRADE ${ }^{I}$ \\ Lucinei Roberto OLIVEIRA ${ }^{\mathrm{II}}$
}

\begin{abstract}
I Acadêmicas do curso de Graduação em Odontologia, Universidade Vale do Rio Verde - UninCor
II $D D S, P h D$. Universidade Vale do Rio Verde - UninCor. Email: lucinei@yahoo.com
\end{abstract}

\begin{abstract}
RESUMO: A doença periodontal (DP) é desencadeada por uma agressão microbiana seguida de uma resposta imunoinflamatória específica com repercussões locais e sistêmicas. Diversos autores consideram a DP como um fator de risco potencial para o desenvolvimento de doenças sistêmicas como acidente vascular cerebral, partos pré-termo, diabetes, aterosclerose e infarto do miocárdio. O estudo dos meios pelos quais a DP pode influenciar uma série de desordens sistêmicas é chamado de medicina periodontal. O biofilme aderido aos dentes pode liberar produtos bacterianos tóxicos biologicamente ativos e o periodonto responde a estas substâncias através da inflamação. Existe um espectro variado de respostas inflamatórias periodontais à agressão microbiana, desde uma leve gengivite a uma grave DP destrutiva. Os produtos bacterianos associados a mediadores inflamatórios podem levar o paciente a produzir uma série de respostas sistêmicas. O objetivo desta revisão é discutir as possíveis vias biológicas pelas quais a DP pode influenciar a etiopatogênese de determinadas doenças e desordens sistêmicas.
\end{abstract}

PALAVRAS-CHAVE: Doença Periodontal. Doenças Sistêmicas. Inflamação sistêmica.

\section{Systemic involvement of periodontal disease - what are the consequences?}

\begin{abstract}
The periodontal disease (PD) is triggered by a microbial aggression followed by a specific response with local and systemic repercussions. Several authors consider DP as a potential risk factor for development of systemic diseases such as stroke, diabetes, preterm birth, atherosclerosis and cardiovascular diseases. The study of how PD can influence a number of systemic disorders is called a periodontal medicine. The biofilm attached in teeth can release toxic bacterial products biologically active and the periodontum responds to these substances through the inflammation. There is a varied spectrum of periodontal inflammatory responses to microbial attack, from a slight gingivitis to a severe destructive PD. The bacterial products associated with inflammatory mediators can lead the patient to produce a series of systemic responses. The purpose of this review is to discuss the possible biological pathways by which the PD can influence the etiopathogenesis of some diseases and systemic disorders.
\end{abstract}

KEYWORDS: Periodontal Disease. Systemic Diseases. Systemic inflammation.

\section{INTRODUÇÃO}

A evolução da área da saúde no último século propiciou uma melhora no tratamento de diversos tipos de enfermidades, o que consequentemente proporcionou uma evolução na qualidade e na expectativa de vida dos pacientes. Participando dessa evolução, a Odontologia cada vez mais se insere como um fator de importância fundamental, principalmente quando se trata da melhora da saúde sistêmica do paciente, através de uma atuação em conjunto com uma equipe multidisciplinar, devido ao entendimento de que uma boa saúde começa e se mantém pela boca.

O comprovado aumento na expectativa de vida com o consequente envelhecimento da população, juntamente 
com determinadas influências ambientais, alterações no estilo de vida e alguns hábitos nocivos associados, colaboram atualmente de maneira direta para os altos índices de doenças sistêmicas diagnosticadas na população. A Odontologia integrada ao tratamento multidisciplinar desempenha então um papel importante nas diferentes fases terapêuticas das doenças sistêmicas, pois através de intervenções específicas, pode prevenir ou reduzir de forma efetiva complicações oriundas de processos

\section{A Doença Periodontal}

\section{A DP é uma doença inflamatória crônica de etiologia multifatorial,} caracterizada clinicamente pela destruição dos tecidos de suporte do dente. O acúmulo de bactérias periodontopatogênicas na superfície dentária é uma condição necessária para o seu início, sendo estes microorganismos predominantemente gram-negativos e anaeróbicos, originados da placa bacteriana depositada no sulco gengival (VIEIRA, 2008). A etiologia primária da DP deve-se à presença da placa bacteriana (biofilme) que se acumula nos tecidos dentários. $\mathrm{O}$ estabelecimento e o crescimento do biofilme a partir do sulco gengival resulta em um aprofundamento patológico do sulco gengival através da migração apical do epitélio juncional, destruição das fibras conjuntivas do ligamento periodontal e do osso alveolar. infecciosos de origem bucal, que podem exacerbar e comprometer a saúde sistêmica dos pacientes quando não tratados (OLIVEIRA et al., 2008).

A possível associação entre doença periodontal (DP) e doenças sistêmicas foi postulada há mais de 100 anos atrás. Muitos estudos foram realizados depois, porém, somente nas últimas décadas tem sido evidenciada uma relação significativa entre ambas.

A DP é uma condição bucal patológica comum na idade adulta. $\mathrm{Na}$ clínica odontológica, uma das maiores causas da perda dentária em adultos e idosos é a DP, que tendo característica crônica e progressiva, nos estágios de idade avançada pode completar sua constante e extensa destruição dos tecidos de sustentação do dente (PAGE, 1998).

A DP é uma das doenças crônicas inflamatórias mais comuns do mundo. As evidências indicam que $35 \%$ a $60 \%$ da populaçao mundial sofre de periodontite moderada. No entanto, a DP grave, que resulta na perda dos tecidos periodontais e dentes, pode ocorrer em cerca de 10 a $30 \%$ de diferentes populações. Em grupos com idades mais avançadas, a DP é responsável por cerca de $35 \%$ de todas as perdas dentárias (BROWN et al., 1989; BECKSTROM et al., 2007; OLIVEIRA et al., 2008). A DP é 
considerada generalizada quando acomete mais de $30 \%$ dos dentes (CARLOS et al., 1986).

Apesar das duas doenças bucais mais comuns - cárie dentária e DP - serem de natureza infecciosa, a possibilidade de bacteremias nas periodontopatias é maior, devido à proximidade dos agentes infecciosos e de seus subprodutos ao tecido conjuntivo e seus componentes vasculares. Evidências das últimas décadas comprovam que a DP pode levar à disseminação sistêmica das bactérias orais e de mediadores inflamatórios, sendo

\section{A Doença Periodontal e a Aterosclerose}

A aterosclerose é definida como uma doença inflamatória crônica que diminui o calibre de uma artéria. A obstrução progressiva do vaso é desencadeada pela formação de um ateroma, formado por placas focais de tecido adiposo na camada íntima arterial, contendo também células necróticas, cristais de colesterol, macrófagos carregadores de lipídios, proteínas plasmáticas de superfície tais como fibrinogênio e fibrina, células musculares lisas hipertróficas e linfócitos T. A presença de um ateroma deixa o paciente propenso a tromboses, devido a área associada a ele servir de substrato para aumentar a agregação plaquetária e a formação de trombos (SCANNAPIECO et al., 2003). estes mediadores capazes de iniciar ou manter os mecanismos associados ao desenvolvimento de doenças sistêmicas crônicas (KARNOUTSOS et al., 2008).

Este estudo de revisão da literatura tem como objetivo discutir as possíveis vias biológicas pelas quais a DP pode influenciar a etiopatogênese de determinadas doenças sistêmicas. Abaixo serão abordadas as principais doenças e desordens sistêmicas associadas atualmente com a DP nas publicações científicas especializadas.

Há muito tempo, o acúmulo passivo de colesterol na parede arterial foi atribuído ao desenvolvimento da aterosclerose, a partir do qual a contínua deposição de lipídeos levaria à progressão da estenose e à total obstrução das artérias (VIEIRA, 2008). No entanto, evidências atuais introduziram novos conceitos relacionados a mecanismos imunoinflamatórios que teriam efeitos importantes sobre a gênese e o desenvolvimento desta patologia.

A inflamação endotelial é considerada atualmente a base da formação da placa aterosclerótica, e sabe-se que o efeito indireto mediado pela expressão de mediadores inflamatórios locais e sistêmicos, capazes de alterar o metabolismo lipídico e estimular a liberação das proteínas de fase aguda do fígado, pode desencadear e/ou acelerar os processos ateroscleróticos (VIEIRA, 2008). 
A DP e a aterosclerose apresentam em comum diversos e complexos mecanismos etiológicos, como predisposições genéticas, gênero, diabete, e o mais significativo entre todos, que é o tabagismo (SCANNAPIECO et al., 2003; VIEIRA, 2008). Os estudos contemporâneos sugerem a existência de uma relação direta entre o desenvolvimento de lesões ateroscleróticas e os processos infecciosos, onde o principal mecanismo pelo qual as infecções podem contribuir para a aterosclerose seria através do efeito nocivo direto dos agentes infecciosos com suas toxinas ao endotélio, promovendo uma agressão às células endoteliais e contribuindo assim para a progressão do processo inflamatório desencadeador da formação das placas ateroscleróticas e dos trombos (VIEIRA, 2008).

Além disso, um estudo epidemiológico recente apontou a DP associada à dislipidemia, pois os níveis plasmáticos de colesterol total e triglicérides demonstraramse elevados em pacientes com DP (ANGELI et al., 2003). Neste referido estudo, altos níveis de colesterol e triglicérides foram observados em indivíduos com periodontite comparados aos controles saudáveis, sugerindo uma influência da DP também no metabolismo lipídico, e contribuindo assim com os fatores de riscos clássicos da aterosclerose.

\section{A Doença Periodontal e as Doenças}

\section{Cardiovasculares}

A potencial ligação da DP com as doenças cardiovasculares vem sendo investigada há mais de uma década. Existem evidências experimentais e clínicas associando as doenças cardiovasculares com infecções sistêmicas.

Tem sido observado que pacientes diagnosticados com infecções periodontais graves apresentam maior incidência de manifestações clínicas de doença arterial coronária ou de acidente vascular cerebral (AVC) (ANGELI et al., 2003).

O mecanismo da DP como fator de risco para as doenças cardiovasculares pode ser desencadeado através de endotoxinas bacterianas (lipopolissacarídeos [LPS]) liberadas na corrente sanguínea, que por sua vez induzem a formação de mediadores inflamatórios (Tromboxano A2, Interleucinas $1 \beta$ e 6, Proteína C-Reativa [PCR] e Fator de Necrose Tumoral [FNT]), que são produzidos como uma resposta do organismo à qualquer tipo de agressão, iniciando-se assim um processo inflamatório sistêmico.

No entanto, os mecanismos que ligam as doenças cardiovasculares e periodontal ainda não foram totalmente esclarecidos, e as pesquisas tem sugerido que alguns fatores genéticos e ambientais também podem estar envolvidos nesta associação. 
Neste sentido, a possível relação entre as infecções bucais crônicas, principalmente a DP, com eventos tromboembolíticos e ateroscleróticos vem sendo evidenciada em diversas investigações (SCANNAPIECO et al., 2003; ELTER et al., 2004; DIETRICH et al., 2008). A hipótese defendida é que o aumento do número de bactérias na cavidade bucal poderia culminar na penetração dessas e de seus produtos no tecido gengival, provocando uma resposta imunológica intensa com produção de mediadores inflamatórios. Os produtos bacterianos tóxicos, em particular os LPS, podem afetar a parede endotelial dos vasos, o sistema da coagulação sanguínea e a função plaquetária, atuando desta forma como fator de risco para as doenças cardiovasculares e AVCs (SCANNAPIECO et al., 2003; DIETRICH et al., 2008).

O que foi estabelecido até o momento é que a presença das bactérias periodontopatogênicas expõe o hospedeiro a uma variedade de eventos nocivos sistêmicos em consequência dos mediadores inflamatórios liberados, que podem ser traduzidos principalmente como alterações na integridade endotelial, bacteremias e formação de trombos (SCANNAPIECO et al., 2003; DIETRICH et al., 2008).

O estudo de RUTGER et al. (2003) foi um entre muitos no qual foi observado uma estreita correlação entre a DP e o Infarto Agudo do Miocárdio. Este problema pode ser explicado, pelo menos em parte, pelo estudo de ANGELI et al. (2003), onde foi constatado que pacientes com a DP tem o ventrículo esquerdo do coração aumentado, provavelmente devido ao maior esforço que o coração precisaria fazer para conseguir bombear sangue através dos vasos sanguíneos que estão totalmente ou parcialmente obliterados por placa de ateromas. Corroborando com estes achados, outras investigações também encontraram os mesmos resultados (JAIN et al., 2003; ELTER et al., 2004;).

Alguns fenômenos relacionados especificamente com os vasos sanguíneos também puderam ser registrados em outros estudos. PERSSON et al. (2002) observaram, por meio de radiografias, pontos de calcificação da artéria carótida, corroborando com o estudo de AMAR et al. (2003), no qual observou-se uma significativa diminuição da dilatação vascular das coronárias nos pacientes portadores de DP.

\section{A Doença Periodontal e o Diabetes Mellitus}

A influência da alteração metabólica relacionada ao Diabetes Mellitus (DM) na etiopatogênese da DP também vem sendo investigada há anos. Tem sido encontrada uma relação direta entre a presença do DM com um maior risco à DP. Contudo, a relação inversa também vem sendo estudada, e essa relação depende, entre outros fatores, do grau 
de controle do paciente diabético (LOESCHE et al., 1997; BRUNETTI, 2004).

A associação entre ambas doenças torna-se ainda mais visível na própria definição do DM. Segundo a American Diabetes Association, “o DM é um grupo de doenças heterogêneas que afetam primariamente o metabolismo dos carboidratos, mas também dos lipídeos e das proteínas. A hiperglicemia é a principal característica e é resultante dos defeitos na secreção da insulina, da resistência à ação desta ou ambos motivos. A hiperglicemia crônica do DM é tóxica ao organismo e está associada a dano, disfunção e falha em vários órgãos, especialmente aos olhos, rins, nervos, coração e vasos sanguíneos" (ADA, 2008).

O paciente diabético apresenta muitas alterações fisiológicas que diminuem a capacidade imunológica e a resposta inflamatória, aumentando a susceptibilidade às infecções (BANDEIRA et al., 2003; CASTILHO \& RESENDE, 1999). Cerca de $4 \%$ dos pacientes adultos que se submetem ao tratamento odontológico são diabéticos, e dentre as afecções sistêmicas que podem estar presentes nesses pacientes, estão inúmeras alterações bucais, como xerostomia e a DP (BRUNETTI, 2004).

No entanto, a principal evidência que tem sido encontrada na relação da DP com o DM é que um bom controle glicêmico pode contribuir para um tratamento mais favorável da DP e, no sentido inverso, quando ocorre uma estabilização da DP, também pode ser observado um melhor controle glicêmico nos pacientes com DM, principalmente naqueles pacientes com DM do tipo 2 (BASCONESMARTINEZ et al., 2011).

\section{Doença periodontal e partos pré-termo}

A relação entre a DP e a incidência de partos prematuros, cujo nome obstétrico é parto pré-termo (PPT), tem sido cada vez mais investigada e evidenciada nos últimos anos.

A ocorrência do PPT se dá anteriormente à $37^{\mathrm{a}}$ semana completa de gestação, tendo-se como base a data do primeiro dia da última menstruação confiável (DUM), sendo decorrente da ruptura prematura da membrana placentária ou do trabalho de parto prematuro propriamente dito, de acordo com a Organização Mundial de Saúde (OMS, 1991).

Um dos fatores implicados na etiologia do PPT pode ser também a infecção bacteriana disseminada sistemicamente, pois a infecção bacteriana, juntamente com os mediadores inflamatórios sistêmicos liberados - como as prostaglandinas, podem atuar como fatores desencadeadores da ruptura prematura da membrana placentária (SPALLICCI et al., 2000). Desta forma, várias pesquisas tem sido 
realizadas com o intuito de determinar de onde viriam estas infecções.

As investigações realizadas por COLLINS et al. (1994), com inoculação subcutânea em hamsters de uma das principais bactérias relacionadas à DP - a Porphyromonas gengivalis, demonstraram que estas bactérias poderiam influenciar negativamente a gestação, principalmente retardando o crescimento fetal e sendo

\section{Conclusão}

O comprometimento dos dentes e de suas estruturas de suporte são achados clínicos preocupantes nos pacientes afetados por doenças e desordens sistêmicas, podendo resultar em uma substancial queda na qualidade de vida dos pacientes.

Apesar do grande número de estudos clínicos recentes abordando a relação da DP com consequências sistêmicas, a falta de padronização metodológica em relação aos parâmetros indicativos da DP nestas investigações limita conclusões definitivas. responsável pelo baixo peso do recémnascido.

Além disso, OFFENBACHER et al. (1998), ao observarem que as mães de crianças prematuras e com baixo peso ao nascer apresentavam quadros mais graves de DP, propuseram que as infecções bucais poderiam constituir uma fonte significativa de infecção e inflamação durante a gravidez.

Contudo, apesar das evidências encontradas em diversas investigações, o que parece ser um consenso na literatura científica é o fato de que mais avaliações prospectivas são necessárias.

Por fim, tem sido amplamente admitido que, apesar da DP ainda não ser comprovadamente um fator de risco para algumas doenças e desordens sistêmicas, tal fato não diminui a importância de uma boa manutenção da saúde bucal nos pacientes acometidos por esta doença.

\section{REFERÊNCIAS BIBLIOGRÁFICAS}

1. ADA,www.endocrino.org.br/ada2008/ acesso em 23 março de 2012.

2. AMAR S, GOKCE N, MORGAN S, LOUKIDELI M, VAN DYKE TE, VITA JA. Periodontal disease is associated with brachial artery endothelial dysfunction and systemic inflammation. Arterioscler Thromb
Vasc Biol; 23(7):1245-9, 2003.

3. ANGELI F, VERDECCHIA P, PELLEGRINO $\mathrm{C}$ et al. Association between periodontal disease and left ventricle mass in essential hypertension. Hypertension; 41(3):488-92, 2003. 
4. BANDEIRA F. Endocrinologia e diabetes. Rio de Janeiro: Medsi, 2003. 1109p., 2003.

5. BASCONES-MARTINEZ A, MATESANZ-PEREZ P, ESCRIBANOBERMEJO M, GONZÁLEZ-MOLES MÁ, BASCONES-ILUNDAIN J, MEURMAN JH. Periodontal disease and diabetes-Review of the Literature. Med Oral Patol Oral Cir Bucal; 16(6): e722-9, 2011.

6. BECKSTROM BW, HORSLEY SH, SCHEETZ JP et al. Correlation between carotid area calcifications and periodontitis: a retrospective study of digital panoramic radiographic findings in pretreatment cancer patients. Oral Surg Oral Med Oral Pathol Oral Radiol Endod.; 103:359-66, 2007.

7. BROWN LJ, OLIVER RC, LOE H. Periodontal diseases in the U.S. in 1981: prevalence, severity, extent, and role in tooth mortality. J Periodontol.; 60:363-70, 1989. 8. BRUNETTI MC. Periodontia Médica: Uma Abordagem Integrada. Editora: SENAC - São Paulo, p. 23-39, 2004.

9. CARLOS JP, WOLFE MD, KINGMAN A. The extent and severity index: a simple method for use in epidemiologic studies of periodontal disease. J Clin Periodontol. 13(5):500-5, 1986.

10. CASTILHO LS, RESENDE VLS. Profilaxia antibiótica:quem necessita? Rev. CROMG, Belo Horizonte, v. 5, n. 3, p.146150, 1999.

11. COLLINS PD, WEG VB, FACCIOLI LH, WATSON ML, MOQBEL R, WILLIAMS TJ. Eosinophil accumulation induced by human interleukin-8 in the guinea pig in vivo. Immunology 79: 312-318. 1994.

\section{DIETRICH T, JIMENEZ M, KRALL} KAYE EA, VOKONAS PS, GARCIA RI. Age-dependent associations between chronic periodontitis/edentulism and risk of coronary heart disease. Circulation., 117(13):1668-74, 2008.
13. ELTER JR, CHAMPAGNE CME, OFFENBACHER S, BECK JD. Relationship of Periodontal Disease and Tooth Loss to Prevalence of Coronary Heart Disease $\mathbf{J}$ Periodontol;75(6):782-790, 2004.

14. JAIN A, BATISTA EL JR, SERHAN C, STAHL GL, VAN DYKE TE. Role for periodontitis in the progression of lipid deposition in an animal model. Infect Immun.; 71(10): 6012-8, 2003.

15. KARNOUTSOS K, PAPASTERGIOU P, STEFANIDIS S, VAKALOUDI A.

Periodontitis as a risk factor for cardiovascular disease: The role of antiphosphorylcholine and anti-cardiolipin antibodies. Hippokratia; 12, 3: 144-149, 2008.

16. JAIN, A.; BATISTA, E.L.; SERHAN, C.; STAHL, G.L.; VAN DYKE, T.E. Role for periodontitis in the progression of lipid deposition in an animal model. Infect Immun.; 71(10):6012-8, 2003.

17. LOESCHE WJ, POHL A, KARAPETOW F. Plasma lipids and blood glucose in patientis with marginal periodontitis. J Dent Res. 76:408-9, 1997.

18. OFFENBACHER S, JARED HL, O'REILLY PG, WELLS SR, SALVI GE, LAWRENCE HP, SOCRANSKY SS, BECK JD. Potential pathogenic mechanisms of periodontitis associated pregnancy complications. Ann Periodontol; 3(1): 23350, 1998.

19. OLIVEIRA LR, MACEDO LD, FERRARI T, MAMEDE RCM, SABACHUJFI E, ZUCOLOTO S. Ocorrência, extensão e gravidade da doença periodontal em pacientes que serão submetidos à radioterapia elou quimioterapia. Arquivos em Odontologia. $\square \square$ 44(1): 35-40, 2008.

20. Organização Mundial de Saúde (OMS). Levantamento epidemiológico básico de saúde bucal: manual de instruções. $3^{\text {a }}$ ed. São Paulo: Livraria Editora Santos; 1991. 
21. PAGE RC. The pathobiology of periodontal diseases may affect systemic diseases: inversion of a paradigm. Ann Periodontol. 3(1): 108-20, 1998.

22. PERSSON RE; HOLLENDER LG; POWELL VL; MACENTEE M; WYATT CC; KIYAK HA. Assessment of periodontal conditions and systemic disease in older subjects. II. Focus on cardiovascular diseases. J Clin Periodontol; 29(9):803-10, 2002.

23. RUTGER PG, OHLSSON O, PETTERSSON T, RENVERT S. Chronic periodontitis, a significant relationship with acute myocardial infarction Eur Heart J; 24(23):2108-15, 2003.

\section{SCANNAPIECO FA, BUSH RB, PAJU}

S. Associations between periodontal disease and risk for atherosclerosis, cardiovascular disease, and stroke. A systematic review. Ann Periodontol. 8(1):38-53, 2003.

25. SPALLICCI MDB, PERRONI A, ALBUQUERQUE PB. Uso da isoxsuprina na inibição do trabalho de parto prematuro na Clínica Obstétrica do Hospital Universitário da Universidade de São Paulo. Rev Bras Med.; 57: 65-9, 2000.

26. VIEIRA CLZ. Relação entre doenças periodontais e aterosclerose subclínica em indivíduos com hipercolesterolemia familiar. São Paulo, Tese (Doutorado) - Faculdade de Medicina, Universidade de São Paulo, 137 p., 2008. 